\title{
Diskrepanzbasierte Regelung der kontinuierlichen Kristallisation
}

\author{
Discrepancy Based Control of Continuous Crystallization
}

Stefan Palis, Otto-von-Guericke Universität Magdeburg, Achim Kienle, Otto-von-Guericke Universität Magdeburg und Max-Planck-Institut für Dynamik komplexer technischer Systeme

Zusammenfassung Der Beitrag beschreibt einen neuen Regelungsansatz zur Stabilisierung von Kristallisatoren. Es ist bekannt, dass diese in Verbindung mit einer Feinkornauflösung zu Instabilitäten und dem Auftreten von unerwünschten Oszillationen neigen. Modelle für Kristallisatoren bestehen meist aus einer nichtlinearen partiellen Integrodifferentialgleichung, der Populationsbilanz für die Kristallgrößenverteilung, gekoppelt mit einer gewöhnlichen Differentialgleichung zur Beschreibung der flüssigen Phase, was einen rigorosen Reglerentwurf vor erhebliche Probleme stellt. Deshalb wird im Rahmen dieser Arbeit auf ein Stabilitätskonzept zurückgegriffen, welches Aussagen über die Stabilität eines Prozesses bezüglich zweier verallgemeinerter Abstandmaße, den sogenannten Diskrepanzen, erlaubt. $\longrightarrow$ Summary In this contribution a new control approach for stabilization of crystallizers is presented. It is well known that instabilities and undesired oscillations may occur, when operated with fines dissolution. In general, crystallizer models consist of a nonlinear partial integro-differential equation, the population balance for the crystal size distribution, coupled with an ordinary differential equation for the liquid phase. Therefore, rigorous control design is a challenging issue. In order to overcome this problem in this contribution a stability concept is used, which states stability with respect to two generalized distance measures, the so called discrepancies.

Schlagwörter Populationsbilanzen, Stabilisierung, Kristallisation, Stabilität bezüglich zweier Diskrepanzen

Keywords Population balances, stabilization, crystallization, stability with respect to two discrepancies

\section{Einleitung}

Die Kristallisation dient der Gewinnung von Feststoffen aus Lösungen. Hierbei ist die Übersättigung die treibende Kraft für die Keimbildung und das Kristallwachstum. Zur Erzeugung einer Übersättigung kommt es unter anderem durch Verdampfung von Lösungsmittel (Verdampfungskristallisation) oder durch Kühlung (Kühlungskristallisation).

Bei der Herstellung von größeren Produktmengen werden Kristallisatoren oftmals kontinuierlich betrieben. Um dabei eine Anhäufung von zu vielen kleinen Kristallen zu unterdrücken, kann eine Feinkornauflösung verwendet werden. Hierzu werden die in einer Beruhigungszone oben schwebenden kleinen Kristalle ab- gezogen und durch erneutes Erhitzen oder Hinzufügen von Lösungsmittel wieder aufgelöst. Die feststofffreie Lösung kann anschließend dem Prozess wieder zugeführt werden. Es ist allerdings bekannt, dass bei dieser Betriebsweise der gewünschte stationäre Zustand instabil sein kann, was zu unerwünschten Oszillationen in der gesamten Partikelgrößenverteilung führt [1-3;5]. Zur Lösung dieses Problems, d.h. zur Stabilisierung instabiler stationärer Zustände, bietet sich der Entwurf eines Reglers an. Der Prozess wird in aller Regel durch eine nichtlineare partielle Integrodifferentialgleichung, die Populationsbilanz zur Beschreibung der Partikelphase, gekoppelt mit einer gewöhnlichen Differentialgleichung zur Beschreibung der flüssigen Phase modelliert. Für Mo- 
dellgleichungen dieser Art finden sich in der Literatur verschiedene Ansätze:

- Diskretisierung der partiellen Integrodifferentialgleichung und Verwendung eines Verfahrens der endlich dimensionalen Regelungstheorie [3;7].

- Approximation der Partikelgrößenverteilung mit Hilfe charakteristischer Funktionenklassen, z. B. Laguerre Polynom. Anschließend kann unter Verwendung dieses Ansatzes die partielle Integrodifferentialgleichung auf ein endlich dimensionales Momentenmodell reduziert werden, für das ebenfalls ein Verfahren der endlich dimensionalen Regelungstheorie zur Stabilisierung verwendet werden kann [4-6].

- Linearisierung der partiellen Integrodifferentialgleichung und Berechnung einer Übertragungsfunktion. Erfüllt die Übertragungsfunktion gewisse Anforderungen, wie z. B. eine endliche Anzahl instabiler Pole, so können Verfahren der unendlich dimensionalen $H_{\infty}$ Regelung zur Stabilisierung genutzt werden [10;11]

Das Problem bei den ersten beiden Verfahren ist, dass durch das Fehlen einer allgemeinen Lösungstheorie von partiellen Integrodifferentialgleichungen nur schwer Aussagen über die Güte der endlich dimensionalen Modelle getroffen werden können. Eine rigorose Stabilitätsaussage ist daher in aller Regel nicht möglich. Bei der Verwendung des dritten Ansatzes hingegen ist die Stabilität nur in unmittelbarer Umgebung der Ruhelage garantiert. Darüber hinaus erhält man bei der Verwendung des Verfahrens von Foias, Özbay und Tannenbaum $[11 ; 12]$ einen ebenfalls unendlich dimensionalen Regler, der zur Implementierung durch einen endlich dimensionalen approximiert werden muss.

Zur Überwindung dieser Nachteile wird im Rahmen dieses Beitrags ein Ansatz vorgestellt, der eine Stabilisierung im Sinne Lyapunovs bezüglich zweier Diskrepanzen $\rho$ und $\rho_{H}$, d.h. zweier verallgemeinerter Abstände, erlaubt. Der Ansatz wurde erstmalig in der russischen Literatur [20] für Anwendungen aus dem Bereich der Festkörpermechanik vorgeschlagen. Er wurde von den vorliegenden Autoren bereits erfolgreich auf Granulationsprozesse [8] angewendet. Im Rahmen der vorliegenden Arbeit wird gezeigt, dass sich der Ansatz ebenfalls auf verwandte Probleme aus dem Bereich der Kristallisation anwenden lässt.

\section{Modell}

Es wird der in Bild 1 dargestellte Kristaller mit Beruhigungszone und Feinkornabzug, wie er u.a. in [4] beschrieben ist, betrachtet. Hinweise zur verwendeten Notation findet man in Tabelle 1. Die Konzentration $c_{0}$ im Zulauf kann über eine vorgeschaltete Verdünnung eingestellt werden. Der Abzug an feinen Kristallen aus der Beruhigungszone $\dot{n}_{F e i n}$ wird über ein regelbares Pumpensystem realisiert.

Zur Beschreibung der Zusammensetzung der festen Phase kann zweckmäßigerweise die Kristallgrößenver-

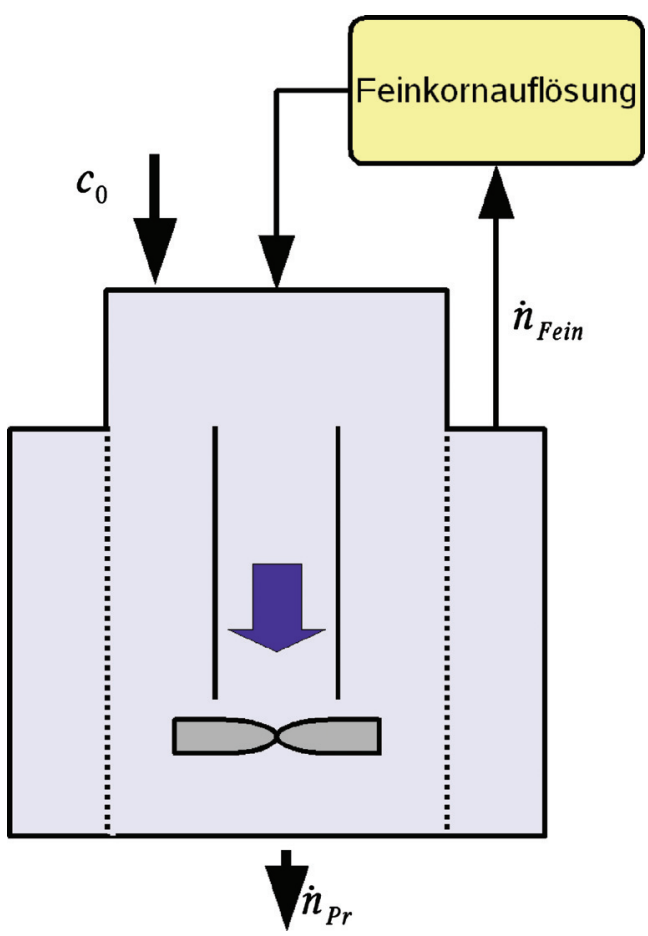

Bild 1 Kristaller mit Feinkornauflösung.

Tabelle 1 Notation.

\begin{tabular}{ll}
\hline$\mu_{i}$ & i-tes Moment, d.h. $\int_{0}^{\infty} L^{i} n d L$ \\
$c_{*}$ & Konzentration \\
$\dot{n}_{*}$ & Partikelfluss \\
$K_{*}$ & Abzugsrate \\
$\varrho_{*}$ & Dichte \\
Indizes & \\
Pr & \\
Fein & Produktpartikel \\
Keime & feine Partikel \\
$s$ & Keime \\
$F$ & Sättigung \\
liq & Feinkornauflösung \\
fest & flüssige Phase \\
ges & feste Phase \\
nom & gesamt \\
& nominell \\
\hline
\end{tabular}

teilung verwendet werden, deren Dynamik kann durch ein Populationsbilanzmodell beschrieben werden. Hierbei handelt es sich um eine nichtlineare partielle Integrodifferentialgleichung, die mit einer gewöhnlichen Differentialgleichung für die flüssige Phase gekoppelt ist.

Unter den vereinfachenden Standardannahmen:

- isothermer Betrieb,

- konstantes Volumen,

- ideale Durchmischung,

- unklassifizierender Abzug,

- kein Abrieb und Bruch 
erhält man eine Populationsbilanz für die Partikelgrößenverteilung $n(t, L)$, wobei $L$ die charakteristische Länge der Einzelkristalle ist.

$\frac{\partial n}{\partial t}=-\frac{\partial G(c) n}{\partial L}-\dot{n}_{P r}-\dot{n}_{F e i n}+\dot{n}_{\text {Keime }}$.

Hierbei ist $G(c)$ die konzentrationsabhängige Wachstumsrate und $\dot{n}_{P r}, \dot{n}_{F e i n}$ und $\dot{n}_{\text {Keime }}$ sind die jeweiligen Raten für Produktabzug, Feinkornauflösung und Keimbildung. Für das Kristallwachstum $G(c)$ wird ein längenunabhängiger, übersättigungsproportionaler Ansatz gewählt:

$G(c)=k_{1}\left(c-c_{s}\right)$.

Der Produktabzug erfolgt unklassierend, d.h. es werden Kristalle unabhängig von ihrer charakteristischen Länge mit der Abzugsrate $K$ entnommen.

$\dot{n}_{P r}=K n$

Da der Abzug des Feinkornes im oberen Bereich der Beruhigungszone erfolgt, kann davon ausgegangen werden, dass lediglich Kristalle mit einer maximalen Länge $L_{F}=1 \mathrm{~mm}$ dem Prozess mit der Abzugsrate $K_{F}$ entzogen werden.

$\dot{n}_{\text {Fein }}=K_{F}\left(1-\sigma\left(L-L_{F}\right)\right) n=K_{F} n_{F} n$

Die Funktion $\sigma$ ist hierbei die Sprungfunktion. Für die Keimbildung wird angenommen, dass Keime der Länge $L=0$ gemäß dem Nukleationsgesetz nach Volmer und Weber [4] erzeugt werden

$\dot{n}_{\text {Keime }}=\left(1-\frac{4}{3} \pi \mu_{3}\right) k_{2} e^{\left(-\frac{k_{3}}{\left(\frac{c}{c_{s}}-1\right)^{2}}\right)}$,

wobei hier $\mu_{3}$ das 3. Moment der Partikelgrößenverteilung $n$ ist, d. h.

$\mu_{3}=\int_{0}^{\infty} L^{3} n d L$

Unter Verwendung der Gleichungen (1)-(5) erhält man daher die Populationsbilanz der Feststoffphase:

$$
\begin{aligned}
\frac{\partial n}{\partial t}= & -\frac{\partial G(c) n}{\partial L}-K n-K_{F} n_{F} n \\
& +\delta(0)\left(1-\frac{4}{3} \pi \mu_{3}\right) k_{2} e^{\left(-\frac{k_{3}}{\left(\frac{c}{c_{s}}-1\right)^{2}}\right)} .
\end{aligned}
$$

In Anbetracht des folgenden Reglerentwurfes wird die Keimbildung nicht, wie üblich, in die Randbedingung geschrieben, sondern über die Deltafunktion direkt in die Populationsbilanz integriert. Die flüssige Phase wird durch die Differentialgleichung für die Lösungsmittelkonzentration beschrieben.

$$
\frac{d c}{d t}=\frac{\left(c_{0}-\varrho\right) K}{\left(1-\frac{4}{3} \pi \mu_{3}\right)}+K(\varrho-c)-\frac{(\varrho-c)}{\left(1-\frac{4}{3} \pi \mu_{3}\right)} \frac{4}{3} \pi \frac{d \mu_{3}}{d t}
$$

\subsection{Stationäre Lösung}

Zur Stabilisierung instabiler stationärer Zustände müssen diese, da sie als Sollwerte in das Regelungsgesetz einfließen, berechnet werden. Im stationären Zustand gilt sowohl $\partial n_{\text {stat }} / \partial t=0$ und damit auch $d \mu_{3, \text { stat }} / d t=0$, als auch $d c_{\text {stat }} / d t=0$. Die Modellgleichungen nehmen daher die folgende Form an:

$$
\begin{aligned}
& \frac{d n_{\text {stat }}}{d L}=-\frac{K+K_{F} n_{F}}{G_{\text {stat }}} n_{\text {stat }}+ \\
& \quad \delta(0) \frac{1-\frac{4}{3} \pi \mu_{3, \text { stat }}}{G_{\text {stat }}} k_{2} e^{\left(-\frac{k_{3}}{\left(\frac{c_{\text {stat }}}{c_{s}}-1\right)^{2}}\right),} \\
& 0=\frac{\left(c_{0}-\varrho\right) K}{\left(1-\frac{4}{3} \pi \mu_{3, \text { stat }}\right)}+K\left(\varrho-c_{\text {stat }}\right) .
\end{aligned}
$$

Die stationäre Lösung der Konzentration erhält man direkt durch umstellen.

$c_{\text {stat }}=\varrho+\frac{c_{0}-\varrho}{1-\frac{4}{3} \pi \mu_{3, \text { stat }}}$

Betrachtet man nun den homogenen Teil der stationären Populationsbilanz:

$\frac{d n_{h}}{d L}=-\frac{K+K_{F} n_{F}}{G_{s t a t}} n_{h}$,

so erhält man für $n_{h}$ :

$n_{h}=C \exp \left(\int_{0}^{L}-\frac{K+K_{F} n_{F}}{G_{\text {stat }}} d L^{\prime}\right)$.

Durch die Methode der Variation der Konstanten erhält man:

$n_{\text {stat }}=\frac{1-\frac{4 / 3}{\pi} \mu_{3, \text { stat }}}{G_{\text {stat }}} e^{-\frac{k_{3}}{\left(\frac{c_{\text {stat }}}{c_{s}}-1\right)^{2}}} e^{\int_{0}^{L}-\frac{K+K_{F} n_{F}}{G_{\text {stat }}} d L^{\prime}}$

bzw. für das 3. Moment der stationären Lösung:

$\mu_{3, \text { stat }}=\int_{0}^{\infty} L^{3} n_{\text {stat }} d L$

Zur numerischen Berechnung der stationären Lösung muss das nichtlineare Gleichungssystem, bestehend aus Gleichung (8), (9) und (10), gelöst werden.

\subsection{Simulationsergebnisse ohne Regelung}

Zur Simulation wird die Populationsbilanz für die Kristallgrößenverteilung Gl. (6) mittels finiter Volumen Methode längendiskretisiert. Es wird hierzu ein örtliches Gitter von 1000 Stützstellen im Gebiet von $0<L<5 \mathrm{~mm}$ gewählt. Zur Simulation werden die in Tabelle 2 angegebenen Parameter [4] verwendet.

Bei der vorliegenden Konfiguration erhält man für eine hohe Abzugsrate in der Feinkornauflösung ein stabiles 
Gesamtsystem, d. h. Abweichungen vom stationären $\mathrm{Zu}$ stand nehmen mit der Zeit ab. In Bild 2 ist dargestellt, wie für die Anfangsverteilung $n_{\text {init }}$ die Oszillationen in der Partikelgrößenverteilung $n$, ihrem 0 . Moment $\mu_{0}$ und der Konzentration $c$ abklingen und ihre stationären Werte annehmen.

Für kleine Abzugsraten verliert die stationäre Lösung ihre Stabilität und es tritt ein stabiler Grenzzyklus auf.

Tabelle 2 Simulationsparameter.

\begin{tabular}{ll}
\hline$\varrho$ & $1,7 \times 10^{3} \frac{\mathrm{kg}}{\mathrm{m}^{3}}$ \\
$c_{s}$ & $980,2 \frac{\mathrm{kg}}{\mathrm{m}^{3}}$ \\
$K$ & 1 \\
$k_{1}$ & $5,065 \times 10^{-2}$ \\
$k_{2}$ & 7,958 \\
$k_{3}$ & $1,217 \times 10^{-3}$ \\
$n_{\text {init }}$ & $7 \times 10^{-3} \exp (-4 L)$
\end{tabular}

Eingangsgrößen ungeregelt

$$
\begin{array}{ll}
c_{0} & 1002 \frac{\mathrm{kg}}{\mathrm{m}^{3}} \\
K_{F} & 0,7 \frac{1}{\mathrm{~h}} \text { bzw. 0,07 } \frac{1}{\mathrm{~h}}
\end{array}
$$
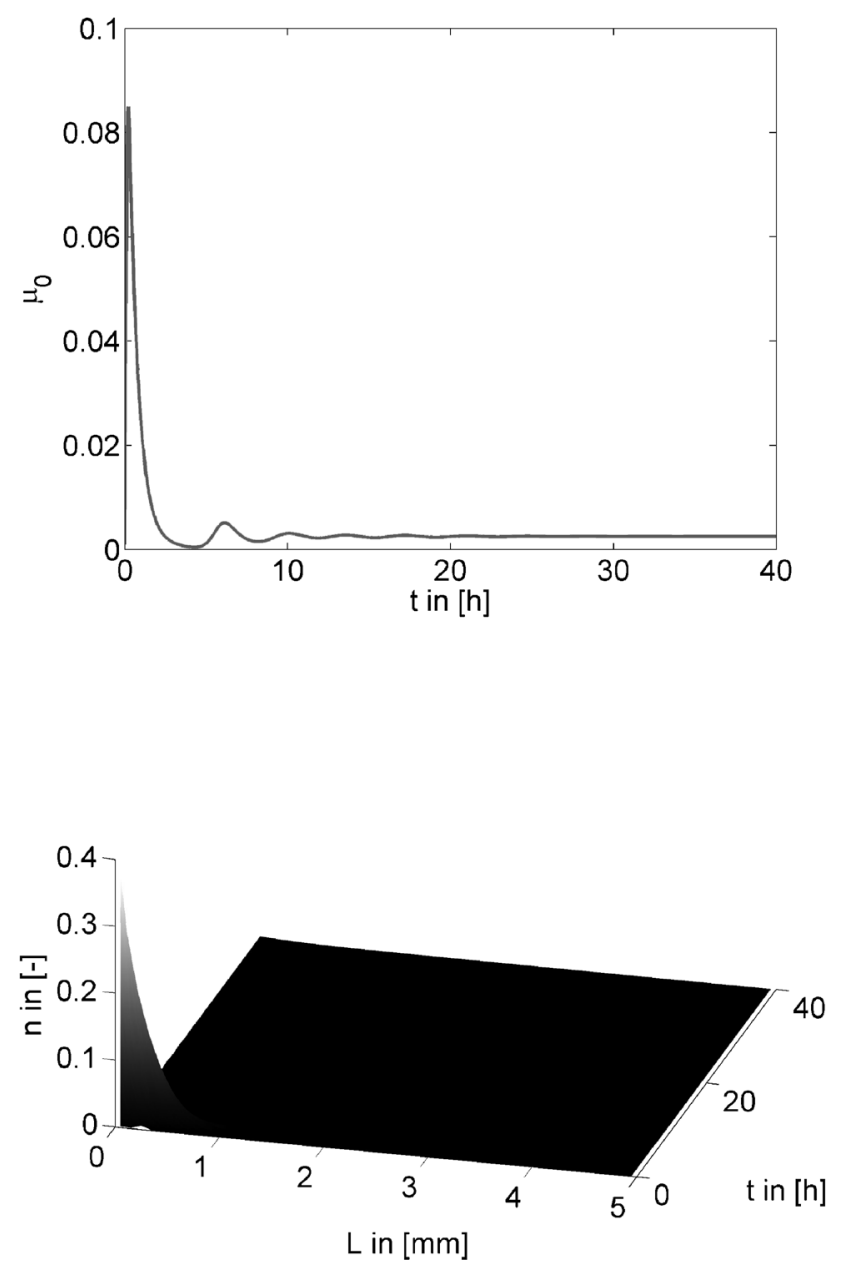

Bild 2 Ungeregeltes Systemverhalten bei $K_{F}=0,7$ und $c_{0}=1002$.
Wie man in Bild 3 erkennt, zeigt der Prozess in diesem Fall ungewünschte Oszillationen in der Anzahlverteilung $n$, ihrem 0 . Moment $\mu_{0}$ und in der Konzentration $c$.

\section{Stabilität und Stabilisierung nichtlinearer Systeme mit verteilten Parametern}

Zur Stabilisierung nichtlinearer Systeme mit verteilten Parametern wurden in den letzten Jahren verschiedenste Methoden entwickelt, die jedoch nahezu alle auf der Lösung der partiellen Differentialgleichung selbst oder des Fehlersystems, d.h. des Systems im geschlossen Regelkreis, basieren. Meist wird dem Gesamtsystem durch einen geeigneten Regler ein Verhalten aufgeprägt, welches sich wieder durch eine partielle Differentialgleichung mit bekannter stabiler Lösung beschreiben läßt. In den Arbeiten von Krstic et al. [13-15] zum Backstepping geschieht dies zum Beispiel durch eine Koordinatentransformation. Während hingegen in den Arbeiten von Bastin et al. [16-18] die Stabilität unter Verwendung der Lösung, gewonnen durch die Methode der Charakteristiken, nachgewiesen wird.

Im vorliegenden Fall der Populationsbilanz (1), einer nichtlinearen partiellen Integrodifferentialgleichung,
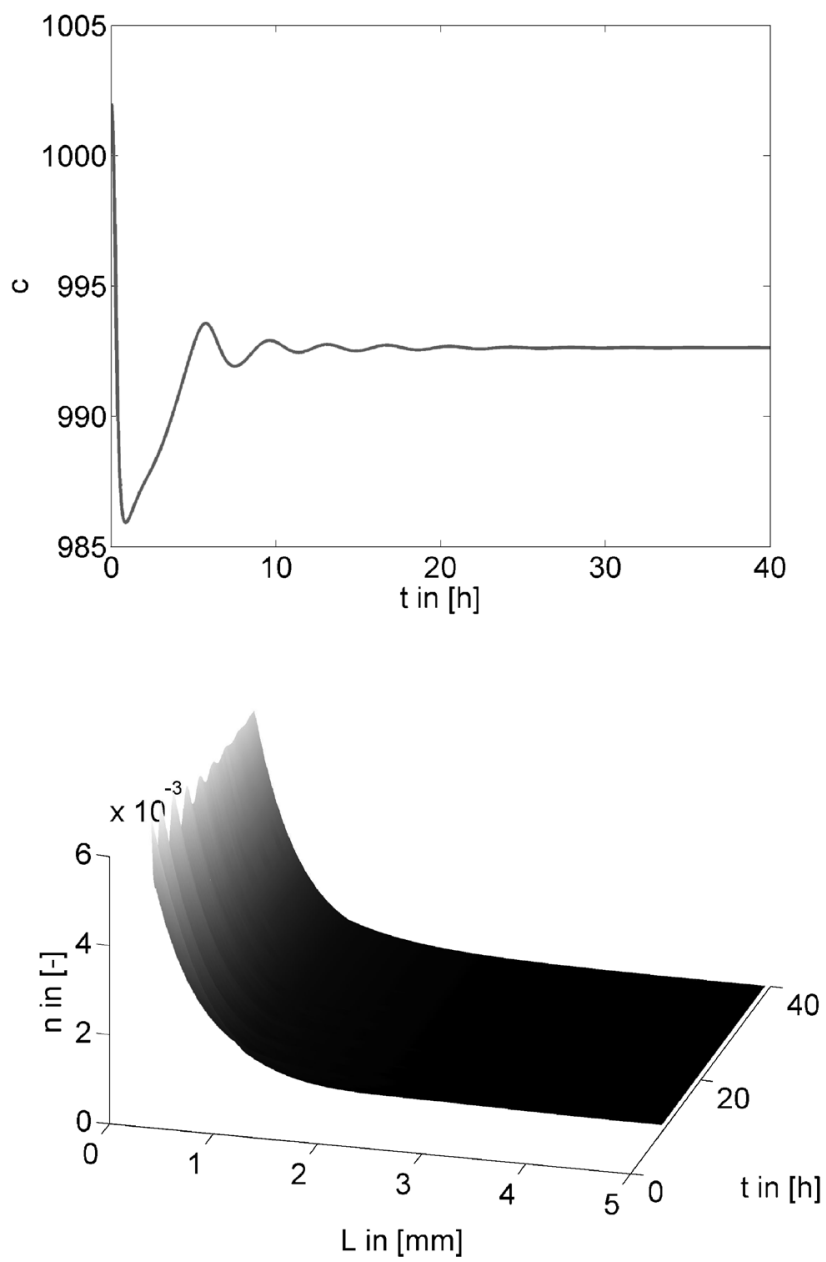

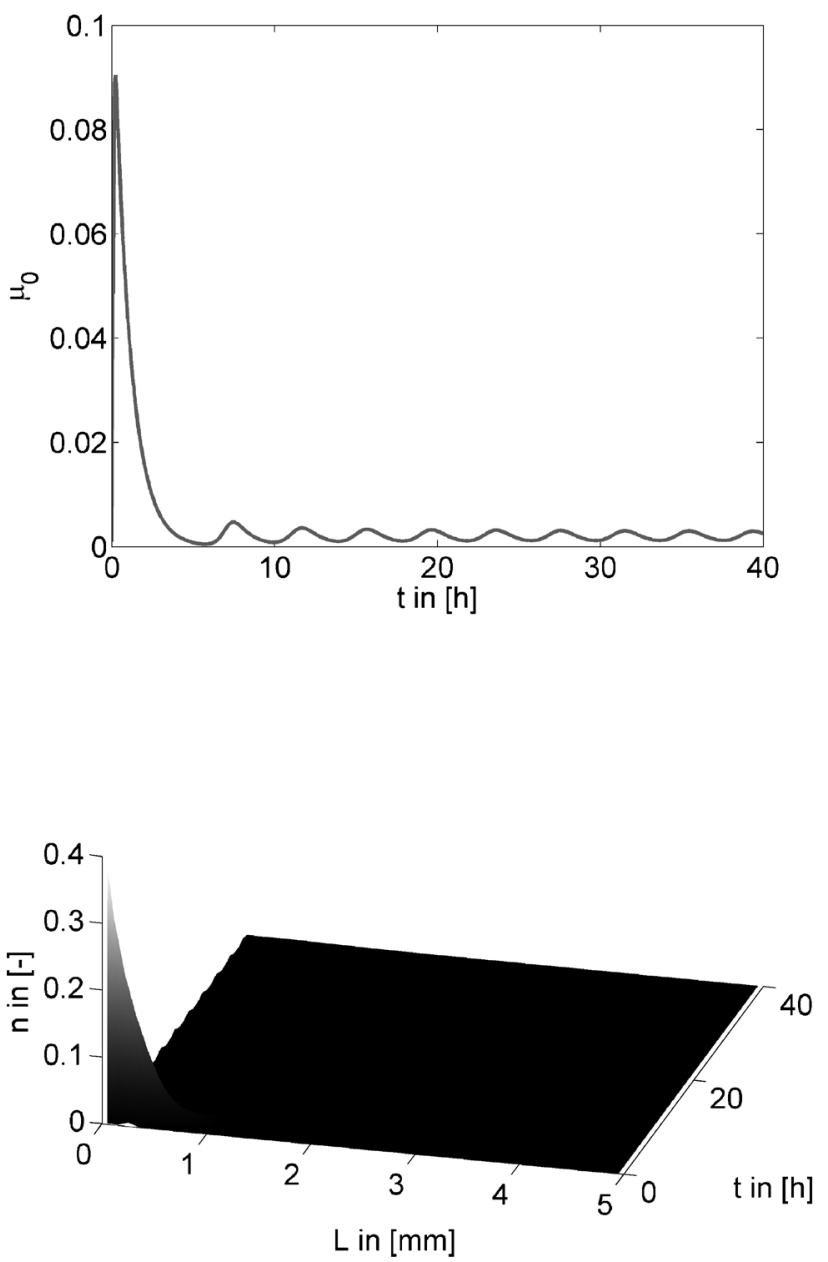

Bild 3 Ungeregeltes Systemverhalten bei $K_{F}=0,07$ und $c_{0}=1002$.

besteht allerdings das Problem, dass auf Grund begrenzter Eingriffsmöglichkeiten und einer weitgehend fehlenden Lösungstheorie, eine Transformation auf ein Fehlersystem mit bekannten Stabilitätseigenschaften im klassischen Sinne nicht möglich ist. Dieses Problem lässt sich durch Einführung eines verallgemeinerten Stabilitätsbegriffes im Sinne zweier verallgemeinerter Abstandsmaße, den sogenannten Diskrepanzen, auf elegante Weise Lösen. Im folgenden seien die wesentlichen Eigenschaften und Aussagen zur Stabilität bezüglich zweier Diskrepanzen in Anlehnung an [2022] zusammengefasst. Hierbei ist $\varphi(., t)$ ein Prozess, d.h. die Lösung des Systems mit verteilten Parametern und damit eine zeitabhängige, verteilte Größe, und $\varphi_{0}=0$ die auf Stabilität zu untersuchende Ruhelage.

Definition 1. Diskrepanz

Eine Diskrepanz ist ein reelles Funktional $\rho=\rho[\varphi(., t), t]$ mit den folgenden Eigenschaften:

1. $\rho(\varphi, t) \geq 0$

2. $\rho(0, t)=0$

3. Für einen beliebigen Prozess $\varphi=\varphi(., t)$ ist das reelle Funktional $\rho(\varphi(., t), t)$ stetig bezüglich $t$.
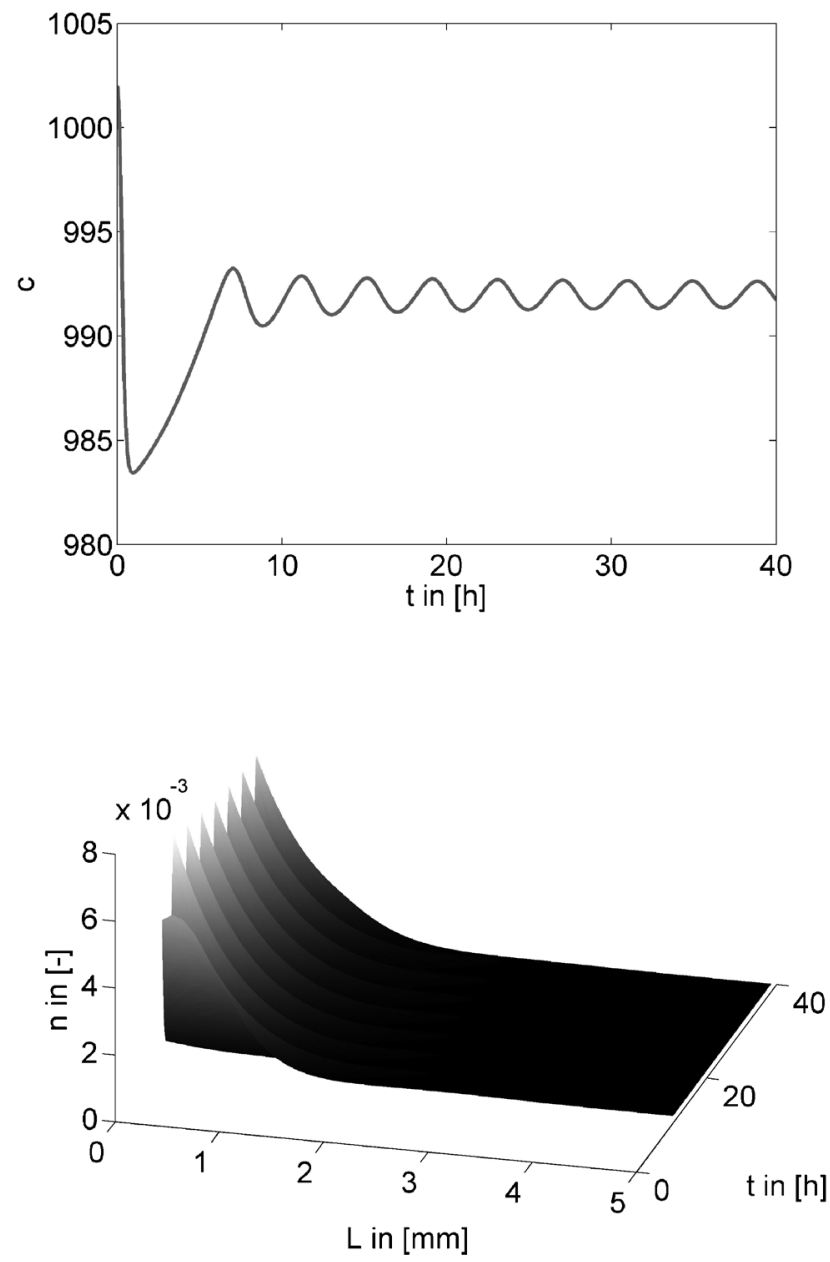

Die Diskrepanz $\rho(\varphi(., t), t)$ beschreibt hierbei den Abstand zwischen dem Prozess $\varphi(., t)$ und der Ruhelage $\varphi_{0}$. Es kann dazu jedes Abstandsmaß mit den oben genannten Eigenschaften verwendet werden. Hierbei ist zu beachten, dass eine Diskrepanz wichtige Eigenschaften einer Metrik nicht erfüllen muss. So wird zum Beispiel bei der Definition der Diskrepanz auf die Symmetrieeigenschaft $d(x, y)=d(y, x)$ und auf die Erfüllung der Dreiecksungleichung $d(x, y) \leq d(x, z)+d(z, y)$ verzichtet. Auch muss eine Diskrepanz nicht die sonst wichtige Eigenschaft der Definitheit erfüllen, d. h. aus dem Verschwinden der Diskrepanz $\rho(\varphi, t)=0$ folgt nicht $\varphi=0$. Damit stellt die Diskrepanz eine Erweiterung der im Rahmen der Stabilitätstheorie sonst üblichen Abstandsmaße wie $L_{p}-$ und Maximumsnormen dar.

Im Rahmen der Stabilitätstheorie im Sinne zweier Diskrepanzen wird zur Bewertung des Abstandes des Anfangszustandes $\varphi(., 0)$ zur Ruhelage $\varphi_{0}$ eine zweite, zeitunabhängige Diskrepanz $\rho_{0}$ eingeführt. An die beiden Diskrepanzen $\rho$ und $\rho_{0}$ wird zusätzlich die Bedingung gestellt, dass die Diskrepanz $\rho(\varphi(., t), t)$ stetig zum Zeitpunkt $t=t_{0}$ bezüglich $\rho_{0}$ an der Stelle $\rho_{0}=0$ ist, d. h. für jedes $\varepsilon>0$ und $t_{0}>0$ existiert ein $\delta\left(\varepsilon, t_{0}\right)>0$, so dass aus $\rho_{0} \leq \delta\left(\varepsilon, t_{0}\right)$ folgt, dass $\rho<\varepsilon$. 
Definition 2. Stabilität bezüglich zweier Diskrepanzen $\rho$ und $\rho_{0}$

Die Ruhelage $\varphi_{0}=0$ ist stabil im Sinne Lyapunovs bezüglich der beiden Diskrepanzen $\rho$ und $\rho_{0}$ für alle $t \geq t_{0}$, wenn für jedes $\varepsilon>0$ und $t_{0} \geq 0$ ein $\delta=\delta\left(\varepsilon, t_{0}\right)>0$ existiert, so dass für jeden Prozess $\varphi(., t)$, für den die Ungleichung $\rho_{0}<$ $\delta\left(\varepsilon, t_{0}\right)$ erfüllt ist, folgt, dass $\rho<\varepsilon$ für alle $t \geq t_{0}$. Wenn zusätzlich gilt, dass $\lim _{t \rightarrow \infty} \rho=0$, dann ist die Ruhelage $\varphi_{0}$ asymptotisch stabil im Sinne Lyapunovs bezüglich der beiden Diskrepanzen $\rho$ und $\rho_{0}$.

Die Grundlage für viele Verfahren der nichtlinearen Regelungstechnik bildet die Stabilitätstheorie nach Lyapunov [23-25]. So kann z. B. anhand einer Regelungs-Lyapunov Funktion unmittelbar ein stabilisierender Regler berechnet werden. Um nun eine ähnlich komfortable Situation für die Stabilität bezüglich zweier Diskrepanzen zu erhalten, muss eine Beziehung zwischen der Existenz eines Lyapunov-Funktionals $V$ mit noch festzulegenden Eigenschaften und der Stabilität bezüglich zweier Diskrepanzen hergestellt werden. Dazu werden im Folgenden die Begriffe der Positivität und positiven Definitheit eines Funktionals bezüglich einer Diskrepanz eingeführt.

Definition 3. Positivität bezüglich einer Diskrepanz $\rho$ Das Funktional $V=V[\varphi, t]$ ist positiv bezüglich der Diskrepanz $\rho$, wenn $V \geq 0$ und $V[0, t]=0$ für alle $\varphi$ mit $\rho(\varphi, t)<\infty$.

Definition 4. Positive Definitheit bezüglich einer Diskrepanz $\rho$

Das Funktional $V=V[\varphi, t]$ ist positiv definit bezüglich der Diskrepanz $\rho$, wenn $V \geq 0$ und $V[0, t]=0$ für alle $\varphi$ mit $\rho(\varphi, t)<\infty$ und für jedes $\varepsilon>0$ ein $\delta=\delta(\varepsilon)>0$ existiert, so dass $V \geq \delta(\varepsilon)$ für alle $\varphi$ mit $\rho[\varphi, t] \geq \varepsilon$.

Die Bedingungen an ein Funktional $V$ zur Garantierung der (asymptotischen) Stabilität bezüglich zweier Diskrepanzen werden in den Sätzen 1 und 2 zusammengefasst.

Satz 1. Der Prozess $\varphi$ mit der Ruhelage $\varphi_{0}=0$ ist stabil bezüglich zweier Diskrepanzen $\rho$ und $\rho_{0}$ genau dann, wenn ein Funktional $V=V[\varphi, t]$ positiv definit bezüglich der Diskrepanz $\rho$, stetig zum Zeitpunkt $t=t_{0}$ bezüglich $\rho_{0}$ an der Stelle $\rho_{0}=0$ und nicht wachsend entlang des Prozesses $\varphi$, d. h. $\dot{V} \leq 0$, existiert.

Satz 2. Der Prozess $\varphi$ mit der Ruhelage $\varphi=0$ ist genau dann asymptotisch stabil bezüglich der zwei Diskrepanzen $\rho$ und $\rho_{0}$, wenn ein Funktional $V=V[\varphi, t]$ positiv definit bezüglich der Diskrepanz $\rho$, stetig zum Zeitpunkt $t=t_{0}$ bezüglich $\rho_{0}$ an der Stelle $\rho_{0}=0$ und nicht wachsend entlang des Prozesses $\varphi$, d. h. $\dot{V} \leq 0$, mit $\lim _{t \rightarrow \infty} V=0$ existiert.

\section{Reglerentwurf}

Im Folgenden soll für die kontinuierliche Kristallisation, beschrieben durch Gl. (6) und (7), eine stabilisierende Regelung entworfen werden. Die dabei interessierenden Größen sind die Konzentration des gelösten Feststoffes c und das nullte Moment der Partikelgrößenverteilung, das der Gesamtanzahl entspricht.

$y=\left(\begin{array}{c}\mu_{0} \\ c\end{array}\right)$

Mögliche Stellgrößen sind sowohl die Abzugsrate für die Feinkornaufösung $K_{F}$, als auch die gelöste Feststoffkonzentration im Zufluss $\mathcal{c}_{0}$.

$u=\left(\begin{array}{c}K_{F} \\ c_{0}\end{array}\right)$

Der Regelfehler wird aus den Abweichungen der Regelgrößen $\left(\mu_{0}\right.$ und $\left.c\right)$ von deren Sollwerten $\left(\mu_{0, d}\right.$ und $\left.c_{d}\right)$ definiert.

$e=\left(\begin{array}{c}\mu_{0, d}-\mu_{0} \\ c_{d}-c\end{array}\right)=\left(\begin{array}{l}e_{1} \\ e_{2}\end{array}\right)$

Weiterhin kann davon ausgegangen werden, dass sowohl die Konzentration $c$, als auch die Partikelgrößenverteilung $n$ gemessen werden können. Zur Erfassung der Partikelgrößenverteilung existieren zahlreiche Messsysteme, wie z. B. FBRM ${ }^{\circledR}$, PVM $^{\circledR}$ oder Parsum Sonde.

Zum Entwurf einer stabilisierenden Regelung findet nun das oben beschriebene Stabilitätskonzept Verwendung. Hierzu wird die Diskrepanz $\rho$ wie folgt definiert:

$\rho=\frac{1}{2}\left(e_{1}^{2}+e_{2}^{2}\right)$.

Offensichtlich erfüllt $\rho$ die oben beschriebenen Anforderungen an eine Diskrepanz. Zu beachten ist an dieser Stelle, dass durch die Verwendung des nullten Momentes die Diskrepanz $\rho$ wichtige Normeigenschaften wie Homogenität, Definitheit und auch die Dreiecksungleichung nicht erfüllt. Zur Gewährleistung der Stetigkeit zum Zeitpunkt $t=t_{0}$ an der Stelle $\rho_{0}=0$ wird $\rho_{0}$ folgendermaßen definiert:

$\rho_{0}=\rho(t=0)$.

Gemäß Satz 2 genügt die Existenz eines entsprechenden Funktionals $V$ zur Absicherung der asymptotischen Stabilität bezüglich der zwei Diskrepanzen $\rho$ und $\rho_{0}$. Es wird daher ein Regelungs-Lyapunov Funktional $V$ wie folgt definiert:

$V=\rho=\frac{1}{2} e^{T} e$.

Zum Erreichen der asymptotischen Stabilität im oben beschriebenen Sinne, müssen die zur Verfügung stehenden Stellgrößen derart gewählt werden, dass die Zeitableitung des Regelungs-Lyapunov Funktionals $\dot{V}$ für alle Zeiten negativ definit ist und nur im Falle $V=0$ verschwindet.

$\dot{V}\left\{\begin{array}{l}<0 \text { für } V \neq 0 \\ =0 \text { für } V=0,\end{array}\right.$

Bildet man die Zeitableitung $\dot{V}$ unter Verwendung der Systemgleichungen (6) und (7), so erhält man die unten aufgeführte Gleichung (12) mit $K_{F}$ und $c_{0}$ als noch 
verbleibende Freiheitsgrade. Hierbei wurde aus Darstellungsgründen auf die Ersetzung von $d \mu_{3} / d t$ durch $\int_{0}^{\infty} L^{3} \partial n / \partial t d L$ und anschließendes Einsetzen der Populationsbilanz (6) verzichtet. Für die Zeitableitung des
Regelungs-Lyapunov Funktionals $\dot{V}$ wird nun gefordert, dass der Klammerausdruck hinter $e_{1}$ gleich $c_{1} e_{1}$ ist und der Klammerausdruck hinter $e_{2}$ gleich $c_{2} e_{2}$ ist, womit sich für $\dot{V}$ folgende Form ergibt:

Zeitableitung des Regelungs-Lyapunov Funktionals $V$

$$
\begin{aligned}
\dot{V} & =e^{T} \dot{e} \\
& =-e^{T}\left(\int_{0}^{\infty}\left[-\frac{\partial G(c) n}{\partial L}-K n-K_{F} n_{F} n+\delta(0)\left(1-\frac{4}{3} \pi \mu_{3}\right) k_{2} \exp \left(-\frac{k_{3}}{\left(\frac{c}{c_{s}}-1\right)^{2}}\right)\right] d L\right) \\
\frac{\left(c_{0}-\varrho\right) K}{\left(1-\frac{4}{3} \pi \mu_{3}\right)}+K(\varrho-c)-\frac{(\varrho-c)}{\left(1-\frac{4}{3} \pi \mu_{3}\right)} \frac{4}{3} \pi \frac{d \mu_{3}}{d t} & -e_{1}\left(-\int_{0}^{\infty}\left(\frac{\partial G(c) n}{\partial L}+K n\right) d L+\left(1-\frac{4}{3} \pi \mu_{3}\right) k_{2} \exp \left(-\frac{k_{3}}{\left(\frac{c}{c_{s}}-1\right)^{2}}\right)-\int_{0}^{L_{F}} n d L K_{F}\right) \\
& -e_{2}\left(\frac{-\varrho K-(\varrho-c) \frac{4}{3} \pi \frac{d \mu_{3}}{d t}}{\left(1-\frac{4}{3} \pi \mu_{3}\right)}+K(\varrho-c)+\frac{K}{\left(1-\frac{4}{3} \pi \mu_{3}\right)} c_{0}\right)
\end{aligned}
$$

Regelgesetz:

$$
\begin{aligned}
& K_{F}=\frac{1}{\int_{0}^{L_{F}} n d L}\left[-c_{1} e_{1}-\int_{0}^{\infty}\left(\frac{\partial G(c) n}{\partial L}+K n\right) d L+\left(1-\frac{4}{3} \pi \mu_{3}\right) k_{2} e^{\left(-\frac{k_{3}}{\left(\frac{c}{c s}-1\right)^{2}}\right)} d L\right] \\
& c_{0}=\frac{\left(1-\frac{4}{3} \pi \mu_{3}\right)}{K}\left[c_{2} e_{2}+\frac{\varrho K+(\varrho-c) \frac{4}{3} \pi \frac{d \mu_{3}}{d t}}{\left(1-\frac{4}{3} \pi \mu_{3}\right)}-K(\varrho-c)\right]
\end{aligned}
$$

$\dot{V}=-c_{1} e_{1}^{2}-c_{2} e_{2}^{2}$.

Diese Forderung kann durch die Wahl der Stellgrößen $K_{F}$ und $c_{0}$ entsprechend Gl. (13) und (14) erfüllt werden. Damit sind die Anforderungen an $\dot{V}$, d.h. Gleichung (11), mit $c_{1}, c_{2}>0$ offensichtlich erfüllt, woraus unmittelbar die asymptotische Stabilität im Sinne Lyapunovs bezüglich der beiden Diskrepanzen $\rho$ und $\rho_{0}$ folgt. Des Weiteren lassen sich über die beiden noch freien positiven Konstanten $c_{1}$ und $c_{2}$ die jeweiligen Abklinggeschwindigkeiten für die entsprechenden Regelfehler einstellen. Für die Zeitableitung des Regelungs-Lyapunov Funktionals gilt außerdem die folgende Abschätzung:

$\dot{V} \leq-c e^{T} e=-2 c V$

$\operatorname{mit} c=\min \left(c_{1}, c_{2}\right)$, woraus die exponentielle Konvergenz von $V$ folgt.

Der vorgestellte Regler besteht aus zwei Komponenten, einer Kompensation der Nichtlinearitäten und einer proportionalen Rückführung des Regelfehlers. Damit ergibt sich die in Bild 4 dargestellte Reglerstruktur.

\subsection{Simulationsergebnisse mit Regelung}

Zur Verifikation des vorgeschlagenen Regelungsansatzes werden die stationären Größen für $\mu_{0}$ und $c_{0}$ im instabilen Bereich als Sollwerte vorgegeben. Hierbei befindet sich im Kristaller, analog zur Simulation ohne Regelung, eine mit der Länge abklingende exponentielle Anfangsverteilung $n_{\text {init }}$. Wie man in Bild 5 sieht, werden sowohl die Regelgrößen, als auch die gesamte Verteilung $n$ stabilisiert. Hierbei konvergiert die Verteilung $n$ gegen ihre stationäre Lösung $n_{\text {stat }}$.

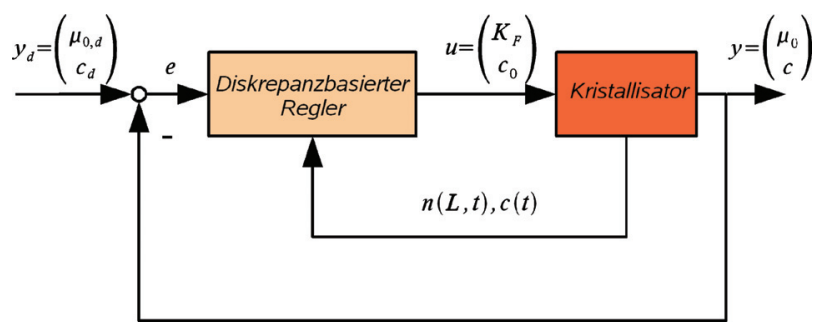

Bild 4 Regelungsstruktur. 

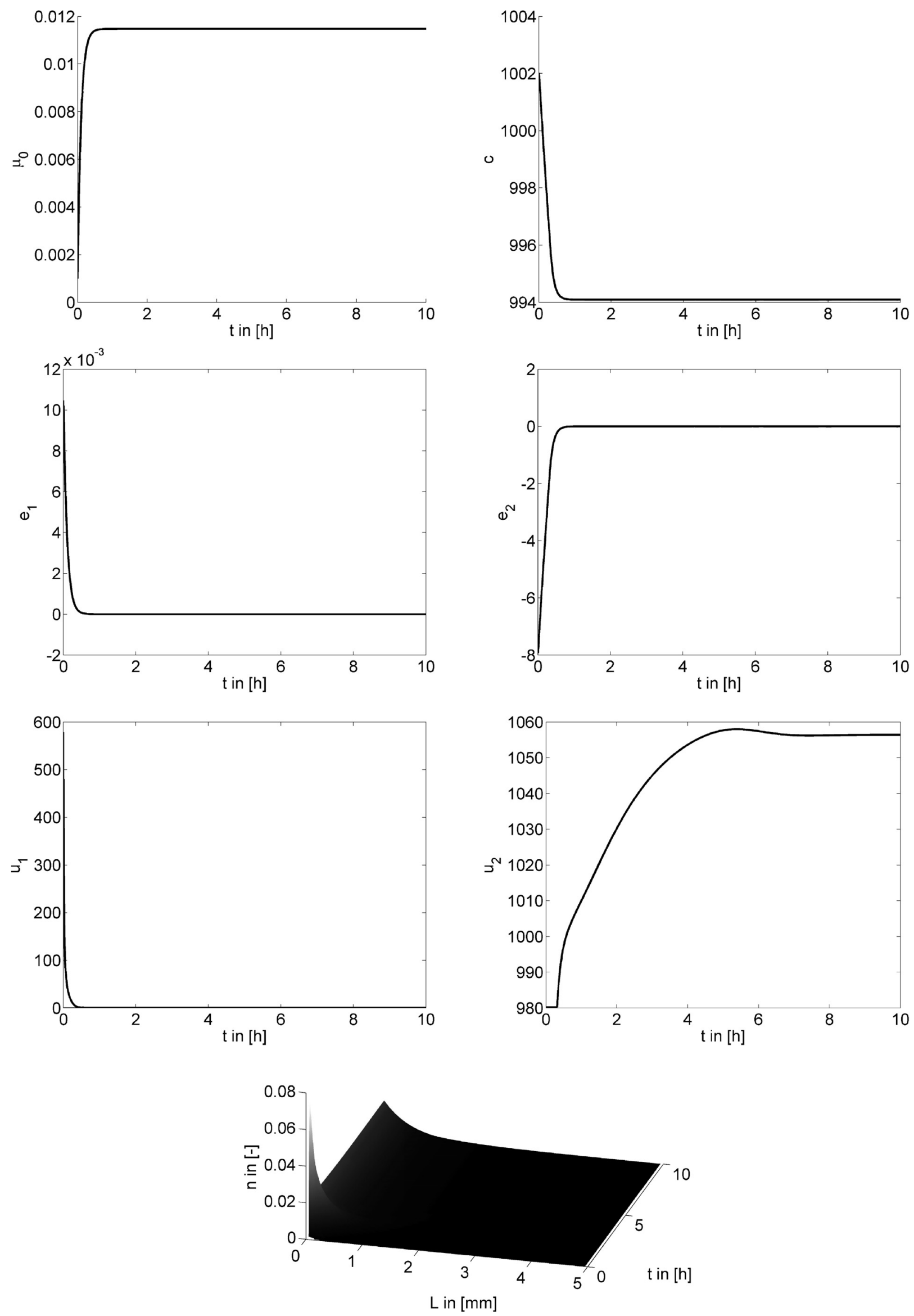

Bild 5 Geregeltes Systemverhalten. 

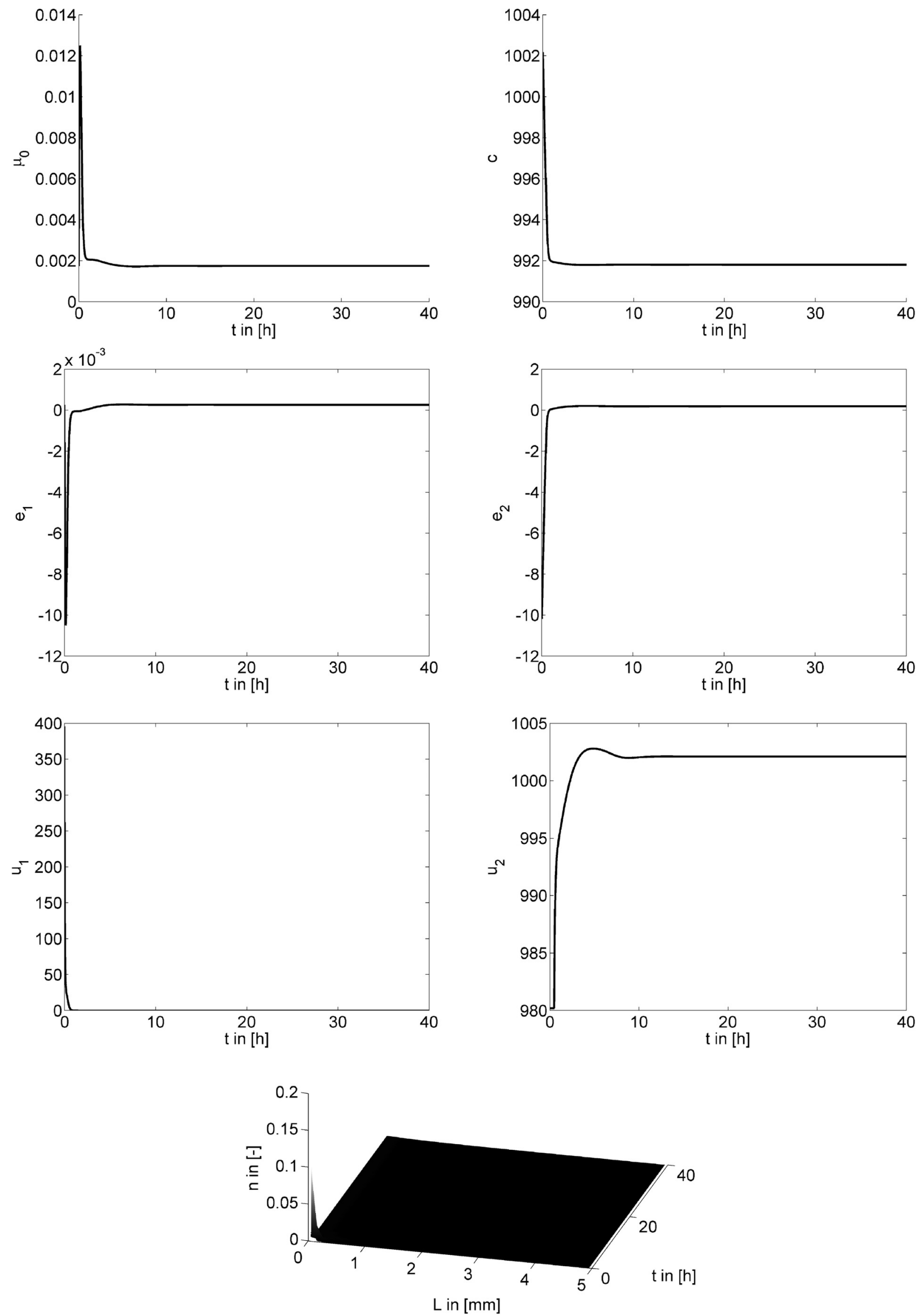

Bild 6 Geregeltes Systemverhalten bei zusätzlichen Unsicherheiten $\left(\varrho=\varrho_{\text {nom }}+10 \% \varrho_{\text {nom }}\right.$ und $\left.k_{2}=k_{2, \text { nom }}+30 \% k_{2, \text { nom }}\right)$. 
Die Konvergenzgeschwindigkeit lässt sich über die beiden Rückführparameter $c_{1}$ und $c_{2}$ vorgeben, wobei bei ihrer Wahl auf das Einhalten von Stellgrößenbeschränkungen zu achten ist.

In Anlehnung an [4] wurden zur Überprüfung der Robustheit des vorgestellten Ansatz parametrische Modellunsicherheiten in $\varrho$ und $k_{2}$ angenommen.

$$
\begin{aligned}
& \varrho=\varrho_{\text {nom }}+10 \% \varrho_{\text {nom }} \\
& k_{2}=k_{2, \text { nom }}+30 \% k_{2, \text { nom }}
\end{aligned}
$$

Wie man in Bild 6 sieht wird auch hier eine Stabilisierung der Regelgrößen und der gesamten Verteilung $n$ erreicht. Allerdings geht die stationäre Genauigkeit verloren.

\section{Zusammenfassung}

Im Rahmen dieser Arbeit wurde ein neuer Regelungsansatz zur Stabilisierung von Kristallisatoren vorgestellt. Die Kernidee ist hierbei, die übliche Forderung nach punktweiser Stabilität der verteilten Größe $n$ durch die Forderung nach Stabilität im Sinne zweier Diskrepanzen abzuschwächen. Diese ist im Allgemeinen nur notwendig, aber nicht hinreichend für punktweise Konvergenz. Punktweise Konvergenz wurde in Simulationsstudien nachgewiesen. Eine noch offene Frage ist unter welchen Bedingungen die Stabilität bezüglich zweier Diskrepanzen die punktweise Konvergenz impliziert.

\section{Literatur}

[1] S. Lei \& R. Shinnar \& S. Katz, The stability and dynamic behavior of a continuous crystallizer with a fines trap, AIChE Journal 17, 1971, pp. 1459-1470.

[2] P.K. Pathath \& A. Kienle, A numerical bifurcation analysis of nonlinear oscillations in crystallization processes, Chem. Eng. Sci. 57, 2002, pp. 4391-4399.

[3] R. Eek, Control and dynamic modelling of industrial suspension crystallizers, Ph.D. thesis, TU Delft, 1995.

[4] T. Chiu \& P. Christofides, Robust Nonlinear Control of a Continuous Crystallizer, Computers and Chemical Engineering Supplement, 1999, pp. S257-S260.

[5] P. Christofides, Model-based control of particulate processes, Kluwer Academic Publ., 2002.

[6] P. Christofides \& N. El-Farra \& M. Li \& P. Mhaskar, Modelbased control of particulate processes, Chem. Eng. Sci. 63, 2008, pp. 1156-1172.

[7] S. Palis \& A. Kienle, Stabilization of continuous fluidized bed spray granulation with external product classification, Chem. Eng. Sci. 70, 2012, pp. 200-209.

[8] S. Palis \& A. Kienle, Stabilization of continuous fluidized bed spray granulation - a Lyapunov approach, 8th IFAC Symposium on Nonlinear Control Systems - NOLCOS, 2010.

[9] R. Curtain \& H. Zwart, An introduction to infinite-dimensional linear systems theory, Springer, 1995.

[10] U. Vollmer \& J. Raisch, $H_{\infty}$-Control a continuous crystallizer, Control Engineering Practice 9, 2001, pp. 837-845.
[11] U. Vollmer \& J. Raisch, Population balance modelling and $H_{\infty^{-}}$ controller design for a crystallization process, Chem. Eng. Sci. 57(20), Oct. 2002, pp. 4401-4414.

[12] C. Foias \& H. Özbay \& A. Tannenbaum, Robust control of infinite dimensional systems: frequency domain methods, Springer, 1996.

[13] A. Balogh \& M. Krstic, Infinite-dimensional backstepping-style feedback transformations for a heat equation with an arbitrary level of instability, European Journal of Control 8, 2002, pp. 165177.

[14] M. Krstic \& A. Smyshlyaev, Adaptive boundary control for unstable parabolic PDEs - Part I: Lyapunov design, IEEE Transactions on Automatic Control 53, 2008, pp. 1575-1591.

[15] A. Smyshlyaev \& E. Cerpa \& M. Krstic, Boundary stabilization of a 1-D wave equation with in-domain anti-damping, SIAM Journal of Control and Optimization 48, 2010, pp. 4014-4031.

[16] J. de Halleux \& C. Prieur \& J-M. Coron \& B. d'Andréa-Novel \& G. Bastin, Boundary feedback control in networks of open channels, Automatica 39(8), 2003, pp. 1365-1376.

[17] J-M. Coron \& B. d'Andréa-Novel \& G. Bastin, A strict Lyapunov function for boundary control of hyperbolic systems of conservation laws, IEEE Transactions on Automatic Control 52(1), 2007, pp. 2-11.

[18] C. Prieur \& J. Winkin \& G. Bastin, „Robust boundary control of systems of conservation laws“, Mathematics of Control, Signals and Systems 20, 2008, pp. 173-197.

[19] V. I. Zubov, Methods of A. M. Lyapunov and their application [in Russisch], Verlag Leningrad LGU, 1957.

[20] A. A. Movchan, Stability of processes with respect to two metrics, Journal of Applied Mathematics and Mechanics 24, 1960, pp. 1506-1524.

[21] T. Sirazetdinov, Stability of systems with distributed parameters [in Russisch], Verlag Nauka, Novosibirsk 1987.

[22] A. Martynjuk \& R. Gutovski, Integral inequalities and stability of motion [in Russisch], Verlag Naukowa Dumka, Kiev, 1979.

[23] M. Krstic \& I. Kanellakopoulos \& P. Kokotovic, Nonlinear and adaptive control design, Wiley-Interscience, 1995.

[24] M. Krstic \& H. Deng, Stabilization of nonlinear uncertain systems, Springer, 1998.

[25] E. D. Sontag, A universal construction of Artstein's theorem on nonlinear stabilization, Systems and Control Letters 13, 1989, 117123.

Manuskripteingang: 17. Mai 2011

Dipl.-Ing. Stefan Palis ist Mitarbeiter am Lehrstuhl Automatisierungstechnik/Modellbildung der Universität Magdeburg. Hauptarbeitsgebiete: Reglerentwurf für Systeme mit verteilten Parametern, adaptive Regelung, nichtlineare Regelung, Regelung von Magnetlagern, Pendelwinkeldämpfung für Drehkräne.

Adresse: Universität Magdeburg, Fakultät für Elektrotechnik, Lehrstuhl für Automatisierungstechnik/Modellbildung, D-39016 Magdeburg, Fax: +49-(0)391-67-11186,

E-Mail: stefan.palis@ovgu.de

Prof. Dr.-Ing. Achim Kienle ist Leiter des Lehrstuhls Automatisierungstechnik/Modellbildung der Universität Magdeburg und Fachgruppenleiter am Max-Planck-Institut für Dynamik komplexer technischer Systeme. Hauptarbeitsgebiete: Modellierung und Regelung chemischer und biologischer Systeme, Energie-Management.

Adresse: siehe oben,

E-Mail: achim.kienle@ovgu.de 